\title{
Detection of the Face using Triplet Method in Messy or Crowdy Video
}

\author{
Pratiksha Choudhari, Priyanka Narke, Vrushali Patil, Pournima Gawhane \\ Department of Computer Engineering, SRCOE, Pune, Maharashtra, India
}

\begin{abstract}
Today India is developing country. All the sectors are influenced by technology, Detection of the Face using Triplet method in messy or crowdy video or an input live video stream for Low enforcement or investigative agency, In Our proposed project we are using a new technique of Face detection with Human object detection the technique is called as deep metric learning.
\end{abstract}

Keywords : Machine Learning, Deep Learning, NN, Python, CCTV

\section{INTRODUCTION}

In Our proposed project we are using a new technique of Face detection with Human object detection the technique is call as deep metric learning. As we understand in deep learning we typically train a network to:

- Accept a single input image

- And output a classification/label for that image

But deep metric learning is different. We are not trying to output a single label or image or coordinates box of objects in an image, we are directly outputting a real-valued vector.

To perform this type of real time data vector and facial recognition in provided image or video we used some external factors to contribute in our detection. These factors are the external dependencies which we are using to build a complete vector graph and plotting facial vectors in order to valued it with inputted video or image to identify the suspect or our target from source image or video.

\section{METHODS AND MATERIAL}

In today's computerized era, every information is being processed by the machine with artificial intelligence and used in many sophisticated applications. Even though many agencies have developed the state-of-the-art security systems, recent terrorist attacks exposed serious weaknesses of sophisticated security systems. Hence various agencies are more serious and motivated to improve security data systems based on body or behavioural characteristics, often called biometrics

[1]. Biometric technologies embrace the identification supported physiological characteristics: face, fingerprints, finger pure mathematics, hand geometry, hand veins, palm, iris, retina, ear, voice and activity traits like gait, signature and keystroke dynamics. 
[2]. Most of the biometric technologies need some voluntary action by the user, i.e. the user must place his hand on a hand-rest for finger printing or hand pure mathematics detection and should stand in a hard and fast position before of a camera for iris or tissue layer identification. However, face recognition will be done passively with none definite action or participation on the two a part of the user, since face pictures will be nonheritable from a distance by a camera, and hence the face recognition system is more appropriate for security and surveillance purposes. Further, knowledge acquisition generally is fraught with issues for alternative biometric techniques that considers hands and fingers. These can be rendered useless if the epidermis tissues are damaged in some way (i.e., bruised or cracked). Expensive equipments square measure needed for the iris and tissue layer identification, and these methods are more sensitive to any body motion. Voice recognition is vulnerable to background noises publicly places and modality fluctuations on a connector or recording. Signatures can be modified or forged. However, facial pictures will be simply obtained with a few of cheap fastened cameras. They cannot be modified or forged, and they are not affected by background sound noise. Face recognition algorithms with acceptable preprocessing of the pictures could catch up on noise, slight variations in orientation, scale and illumination.

\section{III.RESULTS AND DISCUSSION}

Although there square measure blessings of face recognition over different biometric techniques, existing face recognition technology is not able to satisfy the needs. Several challenges square measure there in developing face recognition systems, which are:

\section{A. Illumination Variations}

The direction of illumination within the image, greatly affects the face recognition performance. The variations between constant face pictures because of illumination square measure continuously bigger than variations within the image because of modification in face identity.

\section{B. Frontal vs. Profile}

In a closed circuit television, people are not always facing the cameras. The faces are viewed by some angle. The angle with that the photograph of the individual was in love relevance with the camera affects the face recognition performance drastically.

\section{Expression Variations}

Expression variations in face pictures have an effect on the performance of face recognition. A smiling face, a riant face, a crying face, a sad face, a face with closed eyes, even altitude distinction within the face expression will influence automatic face recognition.

\section{Aging}

Face pictures of a similar individual of one year and fifteen years square measure troublesome to knowledge since face look changes speedily. Images taken by variable a time from five minutes to five years amendment the system accuracy seriously.

\section{E. Occlusions}

It is very difficult to recognize the faces when they are partially occluded. Face pictures in real-world applications could impede because of use of things, like shades, scarf, hands on the face portion, the objects that persons carry, and external sources that occlude the camera view partially. 


\section{IV.SYSTEM ARCHITECTURE}

Figure 1. describes the proposed system architecture which gives the overall idea about developing system. We are trying to develop software which will based on machine learning.

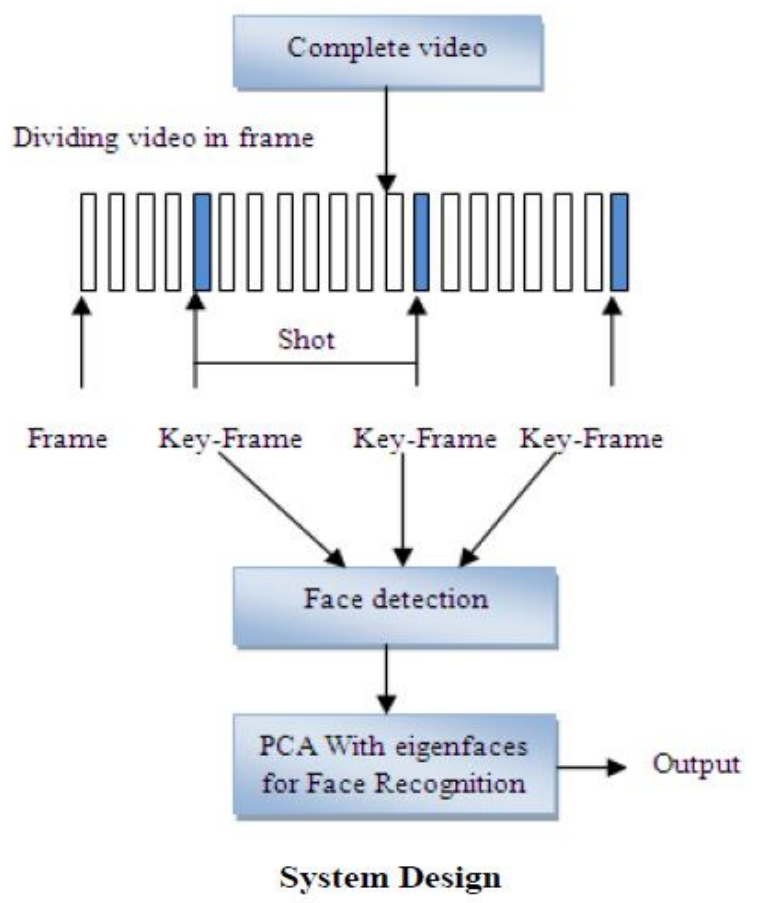

Figure 1: Architecture of Proposed System

Firstly after giving input video and image as a input to a software, it divides complete video into frames. As well as given input image is matches with the trained image dataset.

As the video is played continuously and input image tried to match the face in the video by matching different facial points by using key-frames. Either face get match with face in the input video then it shows result as match found or not found.

Even if the faces in the video are of current date and the inputted image is of $4-5$ years old or it is image of that particular person of Aadhaar card also detect the face in inputted video correctly and gives the correct output result as match found.

In our proposed system the newest technology is used that is triplet method. It overcomes the some drawbacks of previous system. Hence it becomes easier to detect the face using 128-D measurements though the minimal facial points are detected. Also it allows illumination variations as well as occlusions. One of the important aspect is that the image of a particular face is always camera facing but the movement of that face in the video may changes accordingly.

\section{CONCLUSION}

Biometric identification is that the technique of mechanically supportive or distinctive someone by a private attribute or physical characteristic. The term "automatically" suggest that the biometric system should establish or verify an individual's characteristic or attribute promptly with very little or no intervention of the user. Biometric technology was developed for its use in high-level security systems and enforcement markets. The key part of biometric technology is its ability to spot an individual's being and impose security. The aim of this thesis is to develop an automatic face recognition system, which can improve recognition rates for traditional pictures and for pictures with illumination and expression variations.

In order to make it functional on a regular computational CPU we made some functional changes in the dlib facial recognition network, now we can output the feature vector with 128 -d i.e., a list of 128 real-valued numbers that our code will used to quantify the face. While we training network, we use triplets: 


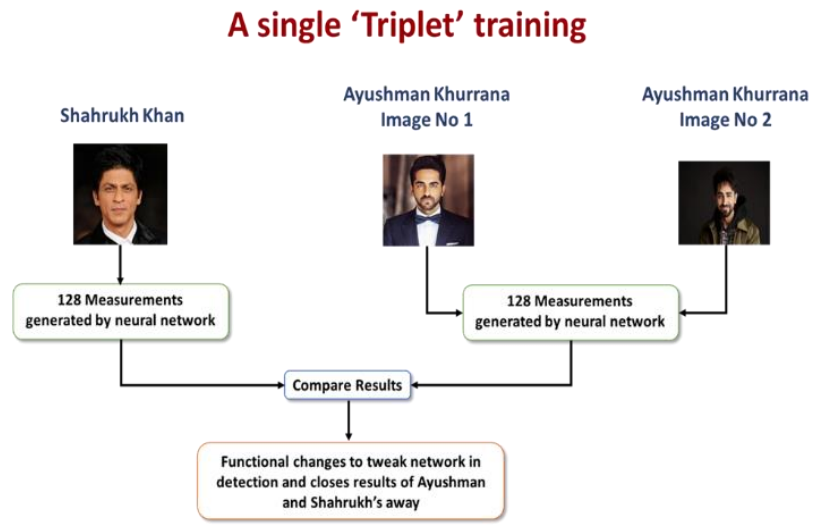

Figure 2: Triplet training

In this demonstration we used three unique faces of popular Cinema Actor. The same functional triplet training consists of the dataset of unique images to train from. With the tradition we used SVM to generate 128-d vector for all images in dataset

- In this we asked SVM to calibrate 128-d for above 3 images where the images of Ayushman are more than 2, hence defined algorithm sense's it as 2 similar faces in dataset.

- Although Shaharukh's image is a random pick from our data set and as not the same as Ayushman

- Our optimized network quantifies the faces and constructs the 128-d embeddings or quantification for each of the images in process

The dataset we used and images we inputted into that are very random and having variety in color, shape, size and even in quality of pixels. This kind of variation is kept to ensure the quality and accuracy in face detection in various condition.

\section{REFERENCES}

[1] Ahmet zdil Metin Mete zbilen A Survey on Comparison of Face Recognition Algorithms IEEE 2015

[2] G. Ramkumar, M. Manikandan - Face Recognition Survey International Journal of
Advances in Science and Technology (IJAST) 2014 ISSN 2348-5426

[3] Sharkas, M. Abou Elenien, Eigenfaces vs. Fisherfaces vs. ICA for Face Recognition; A Comparative Study, 9th International Conference on Signal Processing, 2008, ICSP 2008., 2008, pp. 914919

[4] K. Kim, Intelligent Immigration Control System by Using Passport Recognition and Face Verification, in International Symposium on Neural Networks Chongqing, China, 2005, pp.147-156.

[5] J. N. K. Liu, M. Wang, and B. Feng, iBotGuard: an Internetbased intelligent robot security system using invariant face recognition against intruder, IEEE Transactions on Systems Man And Cybernetics Part C-Applications And Reviews, Vol.35, pp.97-105, 2005.

[6] H. Moon, Biometrics Person Authentication Using Projection- Based Face Recognition System in Verification Scenario, in International Conference on Bioinformatics and its Applications. Hong Kong, China, 2004, pp.207-213.

[7] D. McCullagh, Call It Super Bowl Face Scan 1, in Wired Magazine, 2001.

[8] CNN, Education School face scanner to search for sex offenders. Phoenix, Arizona: The Associated Press, 2003.

[9] P. J. Phillips, H. Moon, P. J. Rauss, and S. A. Rizvi, The FERET Evaluation Methodology for Face Recognition Algorithms, IEEE Transactions on Pattern Analysis and Machine Intelligence, Vol.22, pp.1090-1104, 2000.

[10] T. Choudhry, B. Clarkson, T. Jebara, and A. Pentland, Multimodal person recognition using unconstrained audio and video, in Proceedings, International Conference on Audio and VideoBased Person Authentication, 1999, pp.176181. 
[11] S. L. Wijaya, M. Savvides, and B. V. K. V. Kumar, Illumination-tolerant face verification of low-bit-rate JPEG2000 wavelet images with advanced correlation filters for handheld devices, Applied Optics, Vol.44, pp.655-665, 2005.

[12] E. Acosta, L. Torres, A. Albiol, and E. J. Delp, An automatic face detection and recognition system for video indexing applications, in Proceedings of the IEEE International Conference on Acoustics, Speech and Signal Processing, Vol.4. Orlando, Florida, 2002, pp.3644-3647.

\section{Cite this article as :}

Pratiksha Choudhari, Priyanka Narke, Vrushali Patil, Pournima Gawhane, "Detection of the Face using Triplet Method in Messy or Crowdy Video ", International Journal of Scientific Research in Science and Technology (IJSRST), Online ISSN : 2395-602X, Print ISSN : 2395-6011, Volume 7 Issue 2, pp. 270-274, March-April 2020. Available at doi : https://doi.org/10.32628/IJSRST207237

Journal URL : http://ijsrst.com/IJSRST207237 